\title{
RhoC Peptide Vaccine RV001V
}

National Cancer Institute

\section{Source}

National Cancer Institute. RhoC Peptide Vaccine RV001V. NCI Thesaurus. Code C151937.

A cancer vaccine composed of an immunogenic peptide derived from the Ras homolog family member C (RhoC; Rho-related GT P-binding protein RhoC) that is emulsified in the immunoadjuvant montanide ISA-51, with potential immunomodulating and antineoplastic activities. Upon subcutaneous administration, RhoC peptide vaccine RV001V may stimulate the host immune system to mount a humoral and cytotoxic T-lymphocyte $(\mathrm{CTL})$ response against tumor cells expressing RhoC, which results in tumor cell lysis. RhoC, a tumor-associated antigen (TAA) that is overexpressed in a variety of tumor cell types, is associated with increased metastatic potential. 